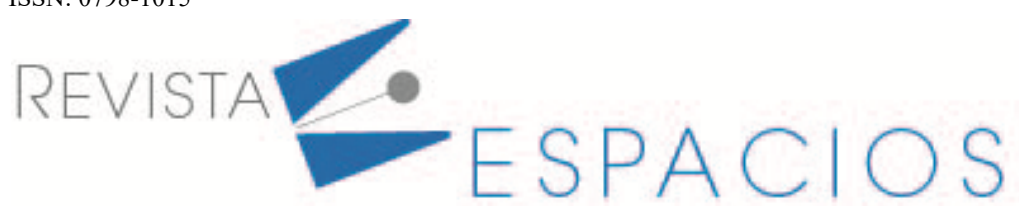

\title{
La comunicación cómo herramienta educativa en la construcción y concienciación ciudadana
}

\section{Communication as an educational tool in construction and citizen awareness}

\author{
ONTANEDA Andrade, Sybel Enrique ${ }^{1}$ \\ SANDOYA Valdiviezo, César Teodomiro ${ }^{2}$ \\ ÁLVAREZ Gomezcoello, Mauricio Esteban ${ }^{3}$ \\ ENRRÍQUEZ Jaya, Isabel María ${ }^{4}$ \\ ONTANEDA Vivanco, Luis Javier ${ }^{5}$
}

\begin{abstract}
Resumen
Analizar la comunicación cómo herramienta educativa en la construcción y concienciación ciudadana, desde la perspectiva de los docentes de la Carrera de Comunicación de la Universidad Nacional de Loja, aplicando el método científico de tipo descriptivo y correlacional, brinda el mayor grado de aplicabilidad para "Conciliar los Derechos Humanos fundamentales de la educación y la comunicación con los deberes y derechos ciudadanos" con el 61,22\% en "Mucho" y el mínimo para "Utilizar la comunicación mediática y las Tics para afianzar las competencias ciudadanas" es de una valía media para los docentes al mostrar los valores en el $19,90 \%$ en "Nada".

Palabras clave: comunicación, herramienta educativa, construcción y concienciación ciudadana.

Abstract

Analyze the communication as an educational tool in the construction and public awareness, from the perspective of the professors of the Communication Career of the National University of Loja, applying the scientific method of descriptive and correlational type, provides the highest degree of applicability to "Reconcile Fundamental Human Rights of education and communication with citizen duties and rights "with 61.22\% in" Much "and the minimum to" Use media communication and ICTs to strengthen citizen competencies "is of average value for teachers when showing values at $19.90 \%$ in "Nothing."

Key Words: Communication, Educational Tool, Construction and Public Awareness.
\end{abstract}

\section{Introducción}

\subsection{Problemas/cuestión}

La comunicación cómo herramienta educativa en la construcción y concienciación ciudadana aún no es muy conocida en la producción de contenidos educativos y gestión democrática de los medios, ya que la mayoría de

\footnotetext{
${ }^{1}$ Carrera de Comunicación. Universidad Nacional de Loja. Licenciado en Comunicación Social. Magister en Comunicación Pública de la Ciencia y la Tecnología.sybel.ontaneda@unl.edu.ec.

${ }^{2}$ Carrera de Comunicación. Universidad Nacional de Loja. Licenciado en Ciencias de la Comunicación Social. Magíster en Desarrollo Comunitario. cesar.sandoya@unl.edu.ec.

${ }^{3}$ Dirección de Comunicación. Universidad Católica de Cuenca. Licenciado en Ciencias de la Información y Comunicación Social, Magister en Gerencia de Marketing, Master en Dirección de Relaciones Públicas y Gabinetes de Comunicación. ideasgrupecuador@gmail.com.

${ }^{4}$ Carrera de Educación Básica e Intercultural Bilingüe. Universidad Nacional de Educación. Licenciada en Ciencias de la Comunicación Social, Magíster en Dirección de Comunicación Empresarial e Institucional. isabel.enrriquez@unae.edu.ec

${ }^{5}$ Carrera de Comunicación. Universidad Nacional de Loja. Licenciado en Ciencias de la Comunicación Social, Máster en Investigación en Comunicación. luis.j.ontaneda@unl.edu.ec.
} 
docentes carecen de capacitación en tecnología actualmente y se resguardan en la falta de recursos y tiempo para poder promover la participación ciudadana, la expresión libre y el derecho a la comunicación responsable. Coartando la posibilidad de acceder a los nuevos ciberespacios educativos porque implican un avance que obliga a los educomunicadores, a potenciar el sentido dialógico, solidario, personal e intercultural.

\subsection{Revisión de la literatura}

\section{La comunicación y educación}

La comunicación y educación se entrelazan en un solo término de "educomunicación" qué es un campo de estudios interdisciplinar y transdisciplinar que aborda, al mismo tiempo, las dimensiones teórico-prácticas de dos disciplinas: la educación y la comunicación. Es también conocida como educación en materia de comunicación, didáctica de los medios, comunicación educativa, alfabetización mediática o pedagogía de la comunicación (Barbas, 2012).

La educomunicación tiene como finalidad la construcción y creación colectiva a través del intercambio simbólico y el flujo de significados, es decir se debe considerar la naturaleza colaborativa y participativa de la Educomunicación; así como también las posibilidades creativas y transformadoras y finalmente los medios y códigos a través de los que se establece el proceso educomunicativo.

De esta forma la educomunicación en conjunto con el pensamiento divergente: (habilidad de ver muchas posibles respuestas a una pregunta), y el aprendizaje significativo, buscan aportar con una metodología alternativa, creativa y dinámica que forje ambientes comunicativos de discusión y deliberación multidisciplinar haciendo énfasis en el poder de la cultura mediática como fortalecimiento de la ciudadanía, en donde jóvenes estudiantes descubran y adquieran instrumentos que les permitan finalmente hacer una crítica argumentativa de su entorno mediático y sociocultural (Montel, 2012).

\section{La comunicación y educación en la formación ciudadana}

La comunicación y educación como se indicó anteriormente se fusionan en "educomunicación" que tiene como finalidad estimular el interés de las personas para que se descubran como productoras de saberes, a partir de la apropiación de la información, se logre su multiplicación y debate ante las dos líneas que conlleva la educomunicacion como son; la información y la comunicación. Sabiendo que el inicio es la adquisición de nuevos conocimientos en base a la lectura sobre algo que se desconoce ante ello es imposible validarla sin la comunicación (Franka, 2012).

La educomunciación trata de evitar que la sociedad de la información se convierta en una sociedad de la ignorancia. Ante la enorme cantidad de información que la sociedad se encuentra sometida a diario surge una actitud de renuncia al conocimiento por desmotivación, rendición, y una tendencia a aceptar de forma tácita la comodidad que proporcionan las visiones tópicas prefabricadas. La falta de capacidad crítica, es la otra cara de la creciente ignorancia. Ante esa paradójica ignorancia apuntada, Moeller (2009: 66) hace hincapié en la urgencia de enseñar a ser autónomos desde la crítica a los estudiantes y profesionales, invitando así a las personas a evaluar lo que leen, oyen y ven, aleccionándoles a notar qué es lo que no se está diciendo y qué es lo que no se muestra. Recalcando que lo anterior es crucial para que el ciudadano ejerza de manera eficaz sus propios derechos ciudadanos y su propio acceso a las oportunidades económicas, políticas y sociales (Gozálvez et al., (2014).

\section{La educomunicación cómo alternativa pedagógica}

La pedagogía es considerada como el arte de saber enseñar donde es fundamental implementar los canales de comunicación adecuados que permitan llevar a cabo el proceso educativo en la actual sociedad tecnológica; la 
misma que logra crear e innovar en la educomunicación, planteando un sin número de retos a los docentes en general, donde el autor Hernández (2010), propone cinco desafíos que se desglosan a continuación:

\section{Desafíos de la educación y comunicación}

- Articular el acceso a la educación con la convivencia social: mediante la educación integral se debe cultivar valores con ética hacia una nueva humanización.

- Desarrollar en las nuevas generaciones las inteligencias intra e interpersonal hacia la responsabilidad ciudadana: fomentar desde las aulas la capacidad de entenderse a sí mismo, reconociendo las propias fortalezas y debilidades, los deseos y los miedos.

- Utilizar la comunicación mediática y las Tics para afianzar las competencias ciudadanas: promulgar como docentes qué la agenda ciudadana no sea concebida por los medios como producto comercial que busca 'rating'.

- Conciliar los Derechos Humanos fundamentales de la educación y la comunicación con los deberes y derechos ciudadanos: Recalcar el derecho humano de recibir y difundir información, expresando con libertad y respeto las opiniones y pensamientos.

- Hacer de las políticas públicas un foro permanente de participación, control y legitimación ciudadana: Legitimar el espacio de participación del poder que reside esencialmente en el pueblo, sin que ello signifique caer en un discurso demagógico, carente de un compromiso serio.

Con la introducción de la tecnología y la comunicación cómo objeto de estudio, se han convertido en un fenómeno educativo del siglo XXI, desde los primeros años del siglo pasado se fomentó el uso del cine con fines educativos y durante la Segunda Guerra Mundial se usaron los medios tecnológicos de la época para adiestrar en el menor del tiempo posible

\section{Las Tics en la educación y comunicación ecuatoriana}

Las TIC actúan como instrumentos de apoyo dentro de la comunicación por medio del correo electrónico o la mensajería instantánea, construyéndose posteriormente en herramientas uni-direccionales dentro de la transmisión de información centrada en el docente hacia una incipiente bi-direccionalidad e inclusive multidireccionalidad; haciendo que la transmisión de información se transforme, durante el proceso comunicativo (Laverde y Vallejo, 2016).

Por otra parte, la Agenda Educativa Digital tiene como finalidad apoyar con equipamiento tecnológico en el campo educativo a fin de disminuir la brecha digital en la educación ecuatoriana, potenciándose mediante los proyectos de la agenda dentro del currículo en línea, como también en las ciencias de la computación, la clase inversa, la educomunicación, el laboratorio audiovisual móvil, entre otros proyectos de innovación existentes (Navarrete y Mendieta, 2018).

\subsection{Justificación}

La comunicación cómo herramienta educativa en la construcción y concienciación ciudadana aspira a dotar a toda persona de competencias expresivas imprescindibles, para su normal desenvolvimiento comunicativo y el desarrollo de la creatividad, centrándose principalmente en la capacitación para el manejo de la tecnología o de los medios, tanto en docentes como estudiantes de todos los niveles educativos y de forma primordial en la educación superior. Surgiendo de esta manera el requerimiento de poder establecer la aplicabilidad de los desafíos de la educación y comunicación cómo herramienta educativa en la construcción y concienciación 
ciudadana, desde la perspectiva docente en las carreras de Comunicación, para poder implantar a sus actores en una dimensión de carácter creativo y transformador con una pedagogía crítica que concibe los procesos educativos, la comunicación, los medios y las tecnologías como herramientas de análisis y de acción para la comprensión y transformación del contexto; que permitan replantear la objetividad de las redes de comunicación actuales y globalizadas con la meta de formar individuos analíticos e investigadores de los datos que receptan a diario.

\subsection{Objetivo}

Determinar la aplicabilidad de los desafíos de la educación y comunicación cómo herramienta educativa en la construcción y concienciación ciudadana, desde la perspectiva docente en la carrera de Comunicación de la Facultad de la Educación, el Arte y la Comunicación de la Universidad Nacional de Loja, mediante la contemplación de desafíos educativos permanentes de la interacción humana.

\section{Metodología}

\subsection{Descripción del contexto y de los participantes}

Los participantes fueron 22 docentes que imparten cátedra en la Carrera de Comunicación de la Facultad de la Educación, el Arte y la Comunicación de la Universidad Nacional de Loja, quienes ayudaron a determinar su perspectiva y la aplicabilidad sobre los desafíos de la educación y comunicación para formar a profesionales que incidan en el cambio e innovación de la comunicación, desarrollando espacios de interacción y diálogo a través de los avances tecnológicos y técnicos, comprometidos e involucrados con el desarrollo y el progreso de la sociedad y responden a las necesidades de la ciudadanía y de los medios con el aprovechamiento de las TIC.

\subsection{Instrumentos}

La elaboración de 1 cuestionario ad hoc, basado en el planteamiento de 5 desafíos de la educación y comunicación del autor Hernández (2010), concertándose una dimensión de en 5 ítems de perspectiva docente, con 3 opciones de elección de acuerdo a la preferencia de cada una de las respuestas, que se basan en los tres niveles intermedios de la escala de Likert según los autores Sánchez et al. (1993), "Poco", "Mucho" y "Nada". A continuación, se muestra el cuestionario resumido (Tabla 1):

\section{Tabla 1}

La comunicación cómo herramienta educativa en la construcción y concienciación ciudadana

\begin{tabular}{l|l|l}
\hline Desafíos de la educación y comunicación (Hernández, 2010). & \multicolumn{1}{c}{ Mucho } \\
\cline { 2 - 3 } (Sánchez et al., 1993). & \multicolumn{1}{c}{ Poco } \\
\hline Articular el acceso a la educación con la convivencia social. & & \\
\hline $\begin{array}{l}\text { Desarrollar en las nuevas generaciones las inteligencias intra e } \\
\text { interpersonal hacia la responsabilidad ciudadana. }\end{array}$ & & \\
\hline $\begin{array}{l}\text { Utilizar la comunicación mediática y las Tics para afianzar las } \\
\text { competencias ciudadanas. }\end{array}$ & & \\
\hline $\begin{array}{l}\text { Conciliar los Derechos Humanos fundamentales de la educación y la } \\
\text { comunicación con los deberes y derechos ciudadanos }\end{array}$ & & \\
\hline $\begin{array}{l}\text { Hacer de las políticas públicas un foro permanente de participación, } \\
\text { control y legitimación ciudadana. }\end{array}$ & & \\
\hline
\end{tabular}

Elaboración propia 


\subsection{Procedimiento}

En la Universidad Nacional de Loja, en la Facultad de la Educación, el Arte y la Comunicación, se procedió a solicitar a los docentes de la Carrera de Comunicación colaboren respondiendo una encuesta en línea vía el correo institucional, para iniciar la recogida de datos, que permitió organizar, clasificar y procesar la información obtenida; para posteriormente analizarla e interpretarla procediendo a valorar y comprobar el objetivo de la investigación.

\section{Resultados}

La educación y comunicación en la aplicabilidad docente muestra muchos retos y metas a nivel de formación ciudadana desde la perspectiva del autor Hernández (2010), plantea 5 desafíos cuyos efectos se describen a continuación:

\subsection{Articular el acceso a la educación con la convivencia social}

Desde la perspectiva docente en la Carrera de Comunicación el "Articular el acceso a la educación con la convivencia social" es fundamental al obtener el 45,58\% en "Mucho", el 35,64\% en "Poco" y el 19,18\% en "Nada"; considerando la importancia del saber convivir en los saberes primordiales de la Unesco en el desarrollo del ser humano, coincidiendo con Hernández (2010) quien afirma que mediante la educación integral se debe cultivar valores con ética hacia una nueva humanización.

Figura 1

Resultados del cuestionario

Desafío 1

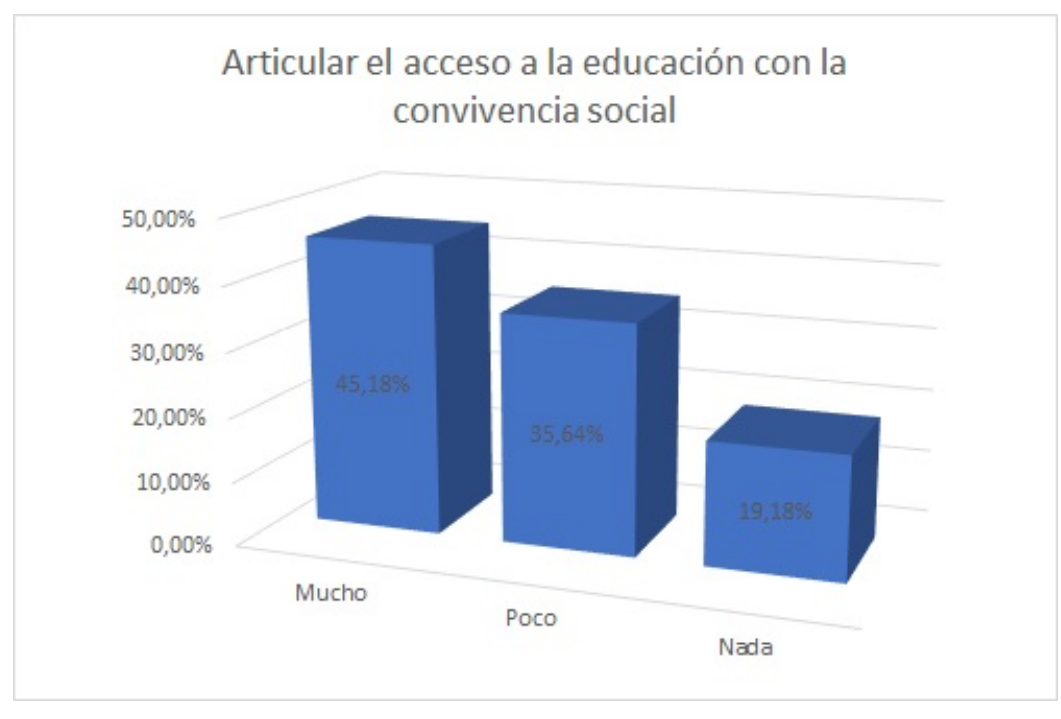

\subsection{Desarrollar en las nuevas generaciones las inteligencias intra e interpersonal hacia la responsabilidad ciudadana}

Los docentes universitarios consideran que "Desarrollar en las nuevas generaciones las inteligencias intra e interpersonal hacia la responsabilidad ciudadana" es un pilar fundamental en el desempeño de un profesional en Comunicación, por esto se logró precisar el 50,31\% en "Mucho", el 40,14\% en "Poco" y el 9,55\% en "Nada" en la aplicabilidad áulica de esta Carrera. Corroborando con los autores Gozálvez y Contreras Pulido (2014), quienes recomiendan que el ciudadano ejerza de manera eficaz sus propios derechos ciudadanos y su propio acceso a las oportunidades económicas, políticas y sociales para potenciar la responsabilidad social. 
Figura 2

Resultados del cuestionario

Desafío 2

Desarrollar en las nuevas generaciones las inteligencias intra e interpersonal hacia la responsabilidad ciudadana

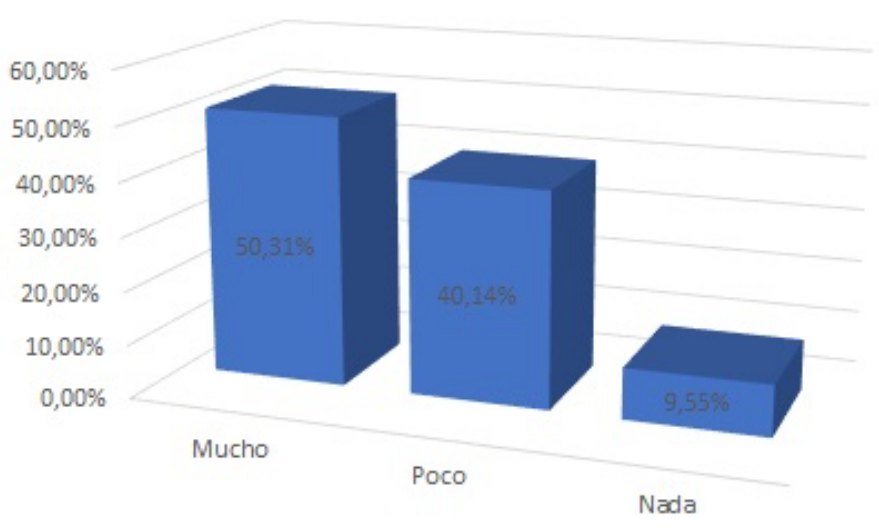

\subsection{Utilizar la comunicación mediática y las Tics para afianzar las competencias ciudadanas}

El poder "Utilizar la comunicación mediática y las Tics para afianzar las competencias ciudadanas" es de una valía media para los docentes al mostrar los valores en el 40,98\% en "Mucho", el 34,12\% en "Poco" y el 19,90\% en "Nada", sin tener presente la verdadera importancia de los medios tecnológicos en la sociedad de la comunicación que se desarrolla en el mundo globalizado donde vivimos.

Estos resultados hacen énfasis en el poder de la cultura mediática como fortalecimiento de la ciudadanía, en donde jóvenes estudiantes descubran y adquieran instrumentos que les permitan finalmente hacer una crítica argumentativa de su entorno mediático y sociocultural (Montel, 2012).

Figura 3

Resultados del cuestionario

Desafío 3

\section{Utilizar la comunicación mediática y las TIC's para}

afianzar las competencias ciudadanas

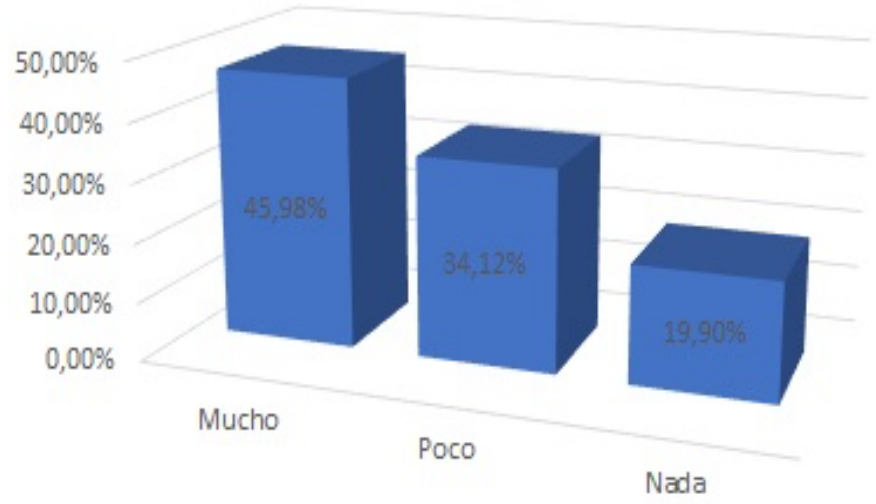




\subsection{Conciliar los Derechos Humanos fundamentales de la educación y la comunicación con los deberes y derechos ciudadanos}

Lograr "Conciliar los Derechos Humanos fundamentales de la educación y la comunicación con los deberes y derechos ciudadanos" desde el enfoque educativo en la Carrera de Comunicación es primordial consiguiendo los más altos puntajes con el 61,22\% en "Mucho", el 24,22\% en "Poco" y el 14,56\% en "Nada", argumentando que los derechos del ser humano son la base principal de la sociedad y la democracia.

Los valores evidenciados en este contexto invitan a citar a Moeller (2009: 66), quien hace hincapié en la urgencia de enseñar a ser autónomos desde la crítica, invitando a las personas a evaluar lo que leen, oyen y ven, y enseñarles a notar qué es lo que no se está diciendo y qué es lo que no se muestra en el trato diario sin perjudicar a ningún individuo en el nombre de la verdad y la justicia al momento de ejercer la profesión de comunicador.

Figura 4

Resultados del cuestionario

Desafío 4

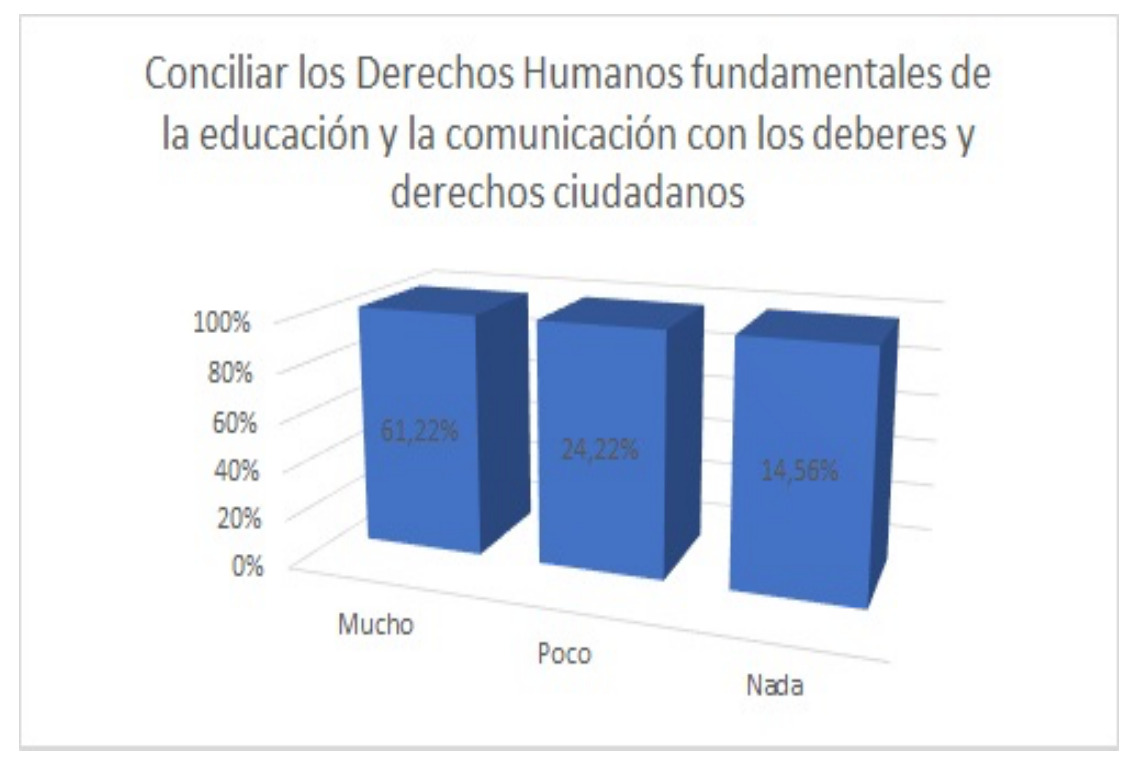

\subsection{Hacer de las políticas públicas un foro permanente de participación, control y legitimación ciudadana}

Los porcentajes alcanzados en el "Hacer de las políticas públicas un foro permanente de participación, control y legitimación ciudadana" muestran una valorización media en la aplicabilidad áulica docente con el 49,36\% en "Mucho", el 36,98\% en "Poco" y el 13,66\% en "Nada", concentrándose en el marco legal vigente para los medios de comunicación en busca de una información real y formativa que beneficie a la comunidad.

Aquí se corrobora lo afirmado por los autores (Laverde y Vallejo, 2016), que el poder ir utilizando herramientas uni-direccionales dentro de la transmisión de información centrada en el docente hacia una incipiente bidireccionalidad e inclusive multi-direccionalidad permiten ir haciendo que la transmisión de información se transforme, durante el proceso comunicativo (Laverde y Vallejo, 2016). 
Figura 5

Resultados del cuestionario

Desafío 5

\section{Hacer de las políticas públicas un foro permanente de participación, control y legitimación ciudadana}

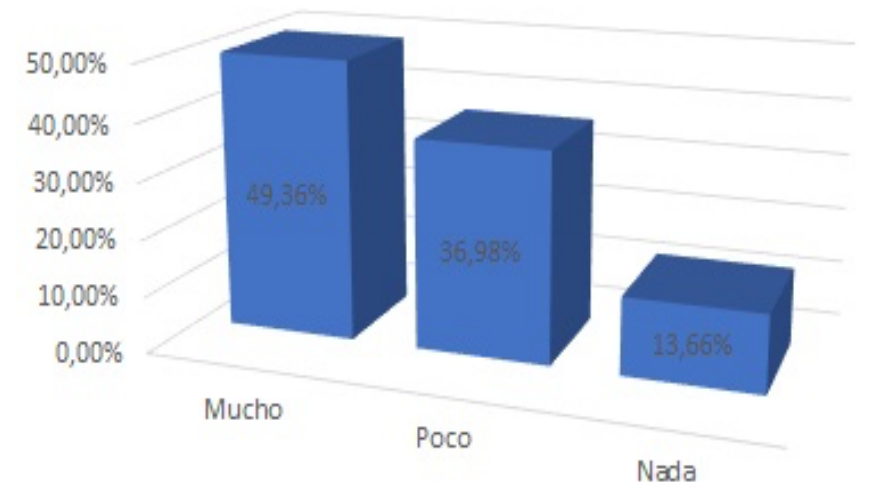

\section{Conclusiones}

Se logró determinar a la comunicación cómo herramienta educativa en la construcción y concienciación ciudadana desde la perspectiva docente en la carrera de Comunicación de la facultad de la Educación, el Arte y la Comunicación de la Universidad Nacional de Loja, con una aplicabilidad docente superior al $50 \%$ en un promedio generalizado.

El poder "Conciliar los Derechos Humanos fundamentales de la educación y la comunicación con los deberes y derechos ciudadanos" obtuvo el mayor porcentaje desde la perspectiva docente con el 61,22\%,", argumentando que los derechos del ser humano son la base principal de la sociedad y la democracia, Moeller (2009: 66).

El mínimo valor de trabajo áulico docente fue para "Utilizar la comunicación mediática y las Tics para afianzar las competencias ciudadanas" es de una valía media para los docentes al mostrar los valores en el 19,90\% en "Nada", haciendo énfasis en el poder de la cultura mediática como fortalecimiento de la ciudadanía, que les permitan hacer una crítica argumentativa de su entorno mediático y sociocultural (Montel, 2012).

\section{Referencias bibliográficas}

Barbas, Á. (2012). Educomunicación: desarrollo, enfoques y desafíos en un mundo interconectado. Foro de Educación, 10(14), 157-175. Recuperado de http://forodeeducacion.com/ojs/index.php/fde/article/view/22

Franka, E. (2012). TICs: La Educomunicación. Recuperado de: http://ticeducomunicacion.blogspot.com/2012/05/tics-la-educomunicacion.html

Gozálvez, V., y Contreras Pulido, P. (2014). Empoderar a la ciudadanía mediática desde la educomunicación. Comunicar: Revista científica iberoamericana de comunicación y educación, (42), 129-136. https://doi.org/10.3916/C42-2014-12

Hernández, G. D. (2010). Desafíos de la educomunicación y alternativas pedagógicas en la construcción de la ciudadanía. IC Revista Científica de Información y Comunicación, (7). 
Laverde, A. C., y Vallejo, V. A. (2016). La Educomunicación en entornos digitales: Un análisis desde los intercambios de información. Opcion, 32(Special Issue 7), 461-479.

Montel, C. (2012). La Educomunicación: Una estrategia pedagógica de ciudadanía critica. Recuperado 16 de noviembre de 2019, de http://tic-educomunicacion.blogspot.com/2012/05/tics-la-educomunicacion.html

Navarrete, G., y Mendieta, C. (2018). Las Tic Y La Educación Ecuatoriana En Tiempos De Internet: Breve Análisis. Espirales, 2(15), 123-136.

Sánchez et al. (1993). Psicología social. Madrid: McGraw-Hill.

Esta obra está bajo una Licencia Creative Commons

Attribución-NoCommercial 4.0 International

\section{(cc) EY-NC}

